\title{
Molecular subtypes of triple-negative breast cancer in women of different race and ethnicity
}

\author{
Yuan Chun Ding ${ }^{1}$, Linda Steele ${ }^{1}$, Charles Warden ${ }^{2}$, Sharon Wilczynski ${ }^{3}$, Joanne \\ Mortimer $^{4}$, Yuan Yuan ${ }^{4}$ and Susan L. Neuhausen ${ }^{1}$ \\ ${ }^{1}$ Department of Population Sciences, Beckman Research Institute of City of Hope, Duarte, CA, USA \\ ${ }^{2}$ Department of Cellular and Molecular Biology, Beckman Research Institute of City of Hope, Duarte, CA, USA \\ ${ }^{3}$ Department of Pathology, City of Hope, Duarte, CA, USA \\ ${ }^{4}$ Department of Medical Oncology, City of Hope, Duarte, CA, USA \\ Correspondence to: Susan L. Neuhausen, email: sneuhausen@coh.org \\ Keywords: triple-negative breast cancer; molecular subtypes; hispanics; asians; african-Americans \\ Received: September 21,2018 Accepted: December 27, $2018 \quad$ Published: January 04, 2019 \\ Copyright: Ding et al. This is an open-access article distributed under the terms of the Creative Commons Attribution License 3.0 \\ (CC BY 3.0), which permits unrestricted use, distribution, and reproduction in any medium, provided the original author and source \\ are credited.
}

\section{ABSTRACT}

Molecular subtypes of triple negative breast cancer (TNBC) are associated with variation in survival and may assist in treatment selection. However, the association of patient race or ethnicity with subtypes of TNBC and clinical outcome has not been addressed. Using nCounter Gene Expression Codesets, we classified TNBCs into subtypes: basal-like immune-activated (BLIA), basal-like immunosuppressed (BLIS), luminal androgen receptor (LAR), and mesenchymal (MES) in 48 Hispanic, 12 African-American, 21 Asian, and 34 White patients. Mean age at diagnosis was significantly associated with subtype, with the youngest mean age (50 years) in MES and the oldest mean age ( 64 years) in LAR $(p<0.0005)$. Subtype was significantly associated with tumor grade $(p=0.0012)$ and positive lymph nodes $(p=0.021)$, with a marginally significant association of tumor stage $(p=0.076)$. In multivariate Cox-proportional hazards modeling, BLIS was associated with worst survival and LAR with best survival. Hispanics had a significantly higher proportion of BLIS (53\%, $p=0.03)$, whereas Asians had a lower proportion of BLIS $(19 \%, p=0.05)$ and a higher proportion of LAR $(38 \%, p=0.06)$ compared to the average proportion across all groups. These differences in proportions of subtype across racial and ethnic groups may explain differences in their outcomes. Determining subtypes of TNBC facilitates understanding of the heterogeneity of the TNBCs and provides a foundation for developing subtype-specific therapies and better predictors of TNBC prognosis for all races and ethnicities.

\section{INTRODUCTION}

Women who present with triple negative breast cancer (TNBC) have worse prognoses than those with other breast cancer subtypes. Defined as breast cancer that lacks expression of estrogen receptor (ER), progesterone receptor (PR), and human epidermal growth factor receptor 2 (HER-2), TNBC is an aggressive histological subtype of breast cancer where women present with high grade, advanced disease. There are limited treatment options and very poor prognosis following progression [1]. Compared to other types of breast cancer, women with TNBC develop recurrent disease early and often to visceral sites. TNBC is sensitive to chemotherapy but responses are short-lived and median survival for those with metastatic disease is only 12 months [2].

TNBC accounts for $10-25 \%$ of all invasive breast cancers depending on race and ethnicity. In a 2010 study of 57,483 breast cancer patients from 17 population-based registries participating in the Surveillance, Epidemiology, and End Results (SEER) program, African-American women were twice as likely and Hispanic women were 
$30 \%$ more likely to be diagnosed with TNBC than nonHispanic Caucasians (hereafter referred to as Whites) [3]. This was consistent with a report from the California Cancer Registry found that women with TNBC were significantly more likely to be African-American or Hispanic and diagnosed under the age of 40 years [4]. The higher incidence of TNBC in women of African ancestry [5] may explain the worse prognosis and higher mortality from breast cancer among African-American [4, 6, 7] compared to White women.

Molecular subtypes of TNBC based on RNA profiling have been shown to be prognostic and predictive of pathological response to neoadjuvant therapy [8-10]. These molecular classifications present an opportunity to improve therapies and therapeutic choices. What has not yet been determined is whether the TNBC molecular subtypes associate with disparities in clinical outcome across race and ethnicity. To date, compared to Whites, few non-White women have been included in studies defining TNBC subtypes, even though both Hispanics and African Americans have a higher proportion of TNBC than Whites [5]. Even within the Cancer Genome Atlas (TCGA) breast cancer set of more than 1000 women, there are few samples from non-White women with TNBC. In this study, we characterized the TNBC subtypes of 48 Hispanic, 12 African-American, 21 East Asian, and 34 White patients and determined the association of these subtypes with clinical outcomes.

\section{RESULTS}

\section{Patient characteristics}

As shown in Table 1, there were no statistically significant differences in characteristics across race and ethnicity. The mean age of patients was 56 years (range 27 to 91). There were 34 Whites, 48 Hispanics, 12 African Americans, and 21 Asians. The majority of women (68\%) presented with self-palpated breast mass (Table 1). Of the women, 38\% of Hispanics, 58\% of African Americans, $32 \%$ of Whites, and $38 \%$ of Asians reported a family history of breast cancer in first- and/or second-degree relatives. There was a marginally statistically significant association $(p=0.06)$ for positive lymph nodes where $43 \%$ of Asians had positive lymph nodes compared to an average of $23 \%$ across all 115 TNBC cases.

\section{Classification of subtypes of TNBC}

Burstein et al. [11] defined four stable TNBC subtypes, basal-like immune-activated (BLIA), basallike immunosuppressed (BLIS), luminal androgen receptor (LAR), and mesenchymal (MES), using the non-negative matrix factorization (NMF) method and determined 80-gene subtype centroid signatures to classify the four NMF-defined TNBC subtypes; we downloaded those data (GSE76124) and replicated the subtype assignment using the Burstein 80-gene centroid signatures. In addition to the 80 genes, we measured expression of 11 genes that identified subtypes of TNBC that were prognostic in a methylome study [12]. Using prediction analysis of microarray (PAM) to optimize the set of genes that could best discriminate the subtypes, we found that the optimal set comprised 77 genes (see Supplementary Table 1 for gene list). Based on a comparison of inconsistencies in subtype calls between the NMF-defined subtypes and subtypes assigned using Burstein's 80-gene centroid signature or comparing the NMF-defined subtypes to subtypes assigned using our 77-gene centroid signatures, we found that the 77-gene signatures had improved accuracy of subtype prediction; there were fewer inconsistencies for the 84 training sample set (inconsistencies of 5 in the 80-gene signatures versus 2 in the 77-gene signatures) and the 114 validation sample set (inconsistencies of 23 in the 80 -gene signatures versus 19 in the 77-gene signatures). Therefore, the analyses results presented below use this 77 -gene signatures. For the 115 samples, we identified the four signatures of BLIA, BLIS, LAR, and MES (Table 2). MES was rare, only being identified in four samples $(3.5 \%)$ compared to $18 \%$ by Burstein et al. [11]. Two cases were unclassified because there was poor assignment to any subtype (both had no centroid correlations $>0.55$ ); these two samples were excluded from further analyses.

\section{Molecular subtypes of TNBCs and association with clinical features and race and ethnicity}

Clinical features for the BLIA, BLIS, MES, and LAR subtypes are shown in Table 2. Mean age at diagnosis was significantly associated with subtype $(p=0.0005)$, with the MES group having the youngest mean age (50 years) and the LAR group having the oldest mean age (64 years) at diagnosis. For tumor characteristics, tumor grade $(p=0.0012)$ and positive lymph nodes $(p=0.021)$ were significantly associated with subtype; there was a marginally significant association of tumor stage and subtype $(p=0.076)$. There was no association of family history or race and ethnicity with the overall distribution of the four subtypes.

\section{Associations of molecular subtypes with recurrence-free and overall survival}

Kaplan-Meier survival curves were constructed. We first examined overall survival and recurrence-free survival for each race and ethnic group (Figure 1A and 1B, respectively). Consistent with reports of others, African Americans had worse overall survival, and Asians had the best survival [13]. We also examined differences in overall survival and recurrence-free survival by stage (Figure 2A and 2B, respectively) and found significant 
Table 1: Characteristics of the $\mathbf{1 1 5}$ participants by race and ethnicity

\begin{tabular}{|c|c|c|c|c|c|c|}
\hline & White & Hispanic & $\begin{array}{c}\text { African } \\
\text { American }\end{array}$ & Asian & Total & $p$-value \\
\hline Number & 34 & 48 & 12 & 21 & 115 & \\
\hline Mean age at diagnosis & 59.7 & 54.7 & 55.8 & 54 & 56.2 & 0.2893 \\
\hline (median; range) & $(60 ; 35-91)$ & $(53 ; 27-77)$ & $(50 ; 37-85)$ & $(52 ; 41-76)$ & $(54 ; 27-91)$ & \\
\hline Family history $^{*}$ & Number (\%) & & & & & \multirow{3}{*}{0.4811} \\
\hline Yes & $11(32.3)$ & $18(37.5)$ & $7(58.3)$ & $8(38.1)$ & $44(38.0)$ & \\
\hline No & $23(67.7)$ & $30(62.5)$ & $5(41.7)$ & $13(61.9)$ & $71(62.0)$ & \\
\hline How first detected & Number (\%) & & & & & \\
\hline Lump felt & $23(67.7)$ & $34(70.8)$ & $7(58.3)$ & $13(61.9)$ & $78(68.0)$ & \multirow{3}{*}{0.6304} \\
\hline Mammogram & $8(23.4)$ & $12(25.0)$ & $5(41.7)$ & $5(23.8)$ & $30(26.0)$ & \\
\hline Other & $3(5.9)$ & $2(4.2)$ & 0 & $3(14.3)$ & $7(6.0)$ & \\
\hline Tumor grade & Number (\%) & & & & & \multirow{4}{*}{0.1383} \\
\hline Grade 2 & $3(8.8)$ & $5(10.4)$ & $3(25.0)$ & $6(28.6)$ & $17(14.8)$ & \\
\hline Grade 3 & $28(82.4)$ & $43(89.6)$ & $9(75.0)$ & $15(71.4)$ & $95(82.6)$ & \\
\hline Missing & $3(8.8)$ & 0 & 0 & 0 & $3(2.6)$ & \\
\hline Tumor stage & Number (\%) & & & & & \\
\hline 1 & $10(29.4)$ & $15(31.3)$ & $4(33.3)$ & $4(19.1)$ & $33(28.7)$ & \multirow{3}{*}{0.8358} \\
\hline 2 & $21(61.8)$ & $28(58.3)$ & $6(50.0)$ & $13(61.8)$ & $68(59.1)$ & \\
\hline 3 & $3(8.8)$ & $5(10.3)$ & $2(16.7)$ & $4(19.1)$ & $14(12.2)$ & \\
\hline \multicolumn{7}{|l|}{ Tumor size } \\
\hline $\mathrm{T} 1$ & $10(29.4)$ & $16(33.3)$ & $6(50.0)$ & $8(38.1)$ & $40(34.8)$ & \multirow{4}{*}{0.4917} \\
\hline $\mathrm{T} 2$ & $20(58.8)$ & $29(60.4)$ & $4(33.3)$ & $10(47.6)$ & $63(54.8)$ & \\
\hline $\mathrm{T} 3$ & $4(11.8)$ & $2(4.2)$ & $2(16.7)$ & $2(9.5)$ & $10(8.7)$ & \\
\hline $\mathrm{T} 4$ & 0 & $1(2.1)$ & 0 & $1(4.8)$ & $2(1.7)$ & \\
\hline Positive lymph nodes & Number (\%) & & & & & \\
\hline Yes & $7(20.6)$ & $7(14.6)$ & $3(25.0)$ & $9(42.9)$ & $26(22.6)$ & \multirow{3}{*}{0.0583} \\
\hline No & $26(76.5)$ & $41(85.4)$ & $8(66.7)$ & $11(52.4)$ & $86(74.8)$ & \\
\hline Missing & $1(2.9)$ & 0 & $1(8.3)$ & $1(4.7)$ & $3(2.6)$ & \\
\hline
\end{tabular}

${ }^{*}$ Family history of breast cancer in first- or second-degree relative.

differences in recurrence-free survival with Stage 1 and 2 having equivalent survival curves and Stage 3 having worse survival ( $p=0.013$; Figure 2B). In the analyses of the subtypes, there were no significant differences in either overall survival or recurrence-free survival, although the BLIA subtype had the best survival of the subtypes (Figure $3 \mathrm{~A}$ and $3 \mathrm{~B}$, respectively). Because there were significant differences in stage by subtype (Table 2) and recurrencefree survival by stage (Figure 2B), we also conducted the analyses of subtype with stage 1 and 2 combined and stage 3 separately (Figure 4A-4D, respectively). There was a significant difference in overall survival by subtype (Logrank test, $p=0.035$ ) among Stage $1 / 2$ cancers (Figure $3 \mathrm{~A}$ ) but no significant differences for recurrence-free survival in Stage 1/2 or for overall or recurrence-free survival for Stage 3 cancers although power was limited.

Cox-proportional hazards modeling was used to assess differences in subtypes for recurrence-free survival and overall survival with the reference being either BLIA subtype or BLIS subtype. Multivariate models were adjusted for age at breast cancer diagnosis, tumor stage, and race and ethnicity. As shown in Table 3, individuals 
Table 2: Clinical features of $\mathbf{1 1 3}$ breast cancer cases by subtype

\begin{tabular}{|c|c|c|c|c|c|}
\hline & BLIA & BLIS & LAR & MES & $P$-value \\
\hline Number & 38 & 46 & 25 & 4 & \multirow{3}{*}{0.0005} \\
\hline Mean Age at diagnosis & 51 & 55 & 64 & 50 & \\
\hline (median; range) & $49 ; 36-85$ & $56 ; 27-77$ & $63 ; 46-91$ & $46 ; 35-76$ & \\
\hline Family history* & Number (\%) & & & & \\
\hline Yes & $18(47.4)$ & $17(37.0)$ & $8(32.0)$ & 0 & \multirow{2}{*}{0.2642} \\
\hline No & $20(52.6)$ & $29(63.0)$ & $14(68.0)$ & $4(100.0)$ & \\
\hline Race and ethnicity & Number $(\%)$ & & & & \\
\hline White & $10(30.3)$ & $12(36.4)$ & $9(27.3)$ & $2(6.1)$ & \multirow{4}{*}{0.1811} \\
\hline Hispanic & $14(29.8)$ & $25(53.2)$ & $6(12.8)$ & $2(4.3)$ & \\
\hline African American & $5(41.7)$ & $5(41.7)$ & $2(16.7)$ & 0 & \\
\hline Asian & $9(42.9)$ & $4(19.1)$ & $8(38.1)$ & 0 & \\
\hline Tumor grade & \multicolumn{2}{|c|}{ Number ( $\%$ of those graded) } & & & \multirow{4}{*}{0.0012} \\
\hline Grade 2 & $2(5.3)$ & $5(10.9)$ & $10(43.5)$ & 0 & \\
\hline Grade 3 & $36(94.7)$ & $41(89.1)$ & $13(56.5)$ & $4(100.0)$ & \\
\hline Missing & 0 & 0 & 2 & 0 & \\
\hline Tumor stage & Number (\%) & & & & \\
\hline 1 & $13(34.2)$ & $12(26.1)$ & $6(24.0)$ & $2(50.0)$ & \multirow{3}{*}{0.0761} \\
\hline 2 & $22(57.9)$ & $31(67.4)$ & $11(44.0)$ & $2(50.0)$ & \\
\hline 3 & $3(7.9)$ & $3(6.2)$ & $8(32.0)$ & 0 & \\
\hline Positive lymph nodes & \multicolumn{2}{|c|}{ Number ( $\%$ of those examined) } & & & \multirow{3}{*}{0.0211} \\
\hline Yes & $10(27.8)$ & $5(10.9)$ & $10(41.7)$ & $1(25.0)$ & \\
\hline No & $26(72.2)$ & $41(89.1)$ & $14(58.3)$ & $3(75.0)$ & \\
\hline Missing & 2 & 0 & 1 & 0 & \\
\hline
\end{tabular}

"Family history of breast cancer in first- or second-degree relatives.

with BLIS had significantly worse $(\mathrm{HR}=11.6, p=0.002)$ and marginally significantly worse $(\mathrm{HR}=2.95, p=0.08)$ recurrence-free survival than those with LAR and BLIA subtypes, respectively. For overall survival, LAR had significantly better survival as compared to both BLIA and BLIS subtypes $(\mathrm{HR}=0.192, p=0.049$, and $\mathrm{HR}=$ $0.073, p=0.001$, respectively). In order to assess whether these differences were associated with race or ethnicity, we examined the distribution of these individual subtypes. Interestingly, for LAR, the percentages varied from $12.8 \%$ in Hispanics to $38.1 \%$ in Asians; for BLIS, percentages ranged from $19.1 \%$ in Asians to $53.2 \%$ in Hispanics (Table 2). Logistic regression models comparing the proportion of the BLIS and the LAR subtypes in each racial and ethnic group to the average proportion of the subtype across all four groups were used to test whether the BLIS and LAR subtypes had the same proportions in each group; Asians were less likely to have the BLIS subtype $(p=0.05)$ and more likely to have the LAR subtype $(p=0.06)$, whereas Hispanics were more likely to have the BLIS subtype $(p=0.03)$ and less likely to have the LAR subtype ( $p=0.10$; Table 4$)$.

\section{DISCUSSION}

This study investigated differences in TNBC subtypes by race and ethnicity. This is particularly important given that Hispanics and Asians are fast-growing populations in the United States; however, they have been largely underrepresented in genomic studies of cancer. Even within TCGA breast cancer set of more than 1000 women, there are few samples from non-White women, and particularly from those with TNBC; in total, there are 8 Asians, 4 Hispanics, and 33 African Americans with TNBC. The lack of knowledge of biological differences underlying disparities in clinical outcomes needs to be addressed. 
The molecular biology of TNBC is heterogeneous. In recent studies using microarray expression data, subtypes of TNBC were identified and associated with differences in treatment response and prognosis. The initial study defined six subtypes: basal-like 1 (BL1), basal-like 2 (BL2), immunomodulatory (IM), mesenchymal (M), mesenchymal stem-like (MSL), and luminal androgen receptor (LAR) subtype $[9,10]$. In a follow-up study, the IM and MSL subtypes were removed after discovering that they originated from infiltrating lymphocytes and tumor-associated stromal cells, respectively, and thus were not true TNBC tumor subtypes [14]. In a more

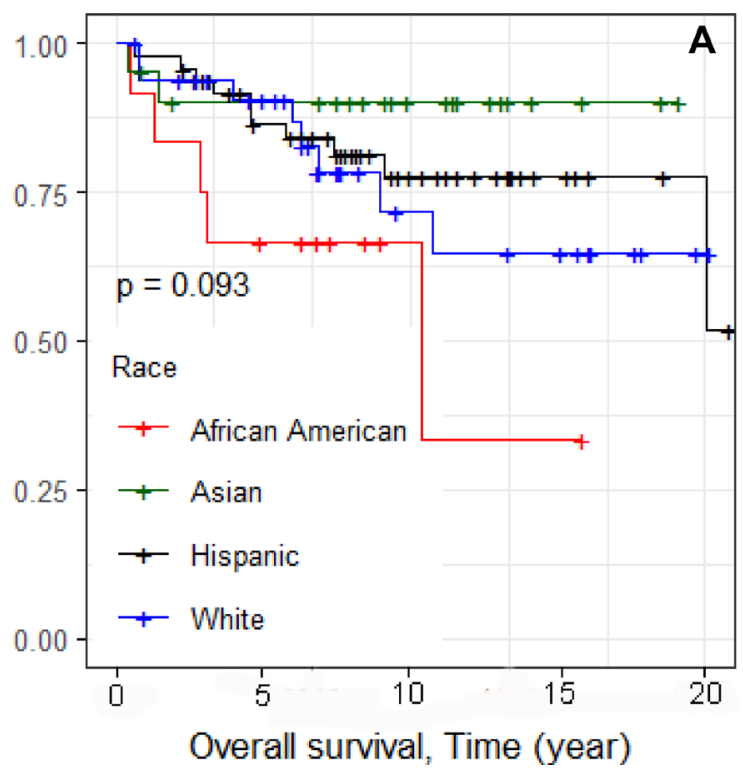

recent study, Burstein et al. [11] defined four subtypes of BLIA, BLIS, MES, and LAR [11]. In a comparison of the distribution of TNBC subtypes between the Lehman and Burstein signatures using the same set of samples, the MES signature of Burstein almost completely overlapped with the IM and MSL subtypes of Lehmann et al. [15]; we found the same result in our comparison of 114 samples (GSE76124) where the MES subtype consisted of 31\% IM and $62 \%$ MSL subtype. Because the MES largely reflects the mixture of stromal or immune cells with epithelial cells, the MES subtype may depend on how carefully epithelial cells were selected from FFPE blocks.

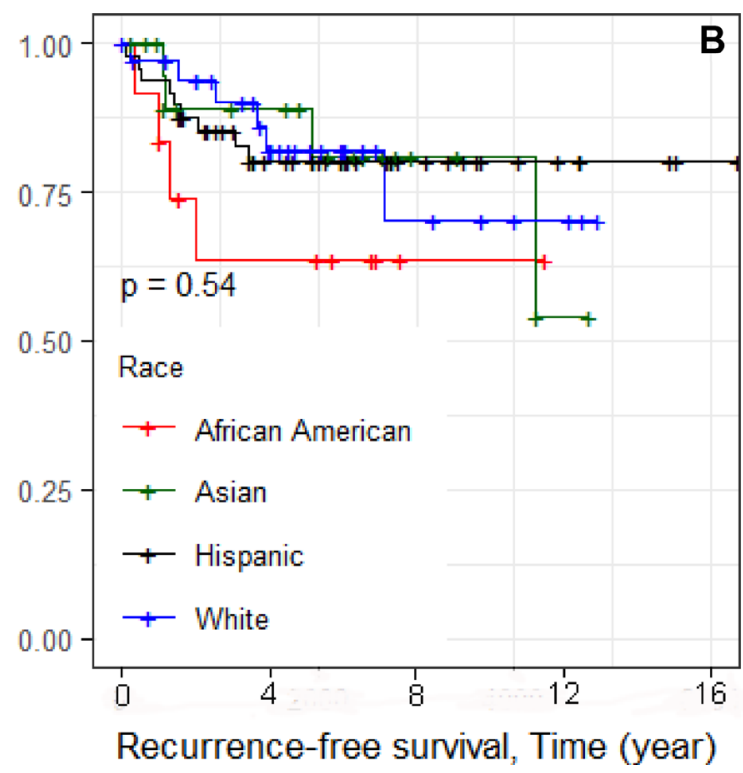

Figure 1: Kaplan-Meier analyses of overall and recurrence-free survival and race/ethnicity for 115 TNBC cases (Figure 1A and 1B, respectively).
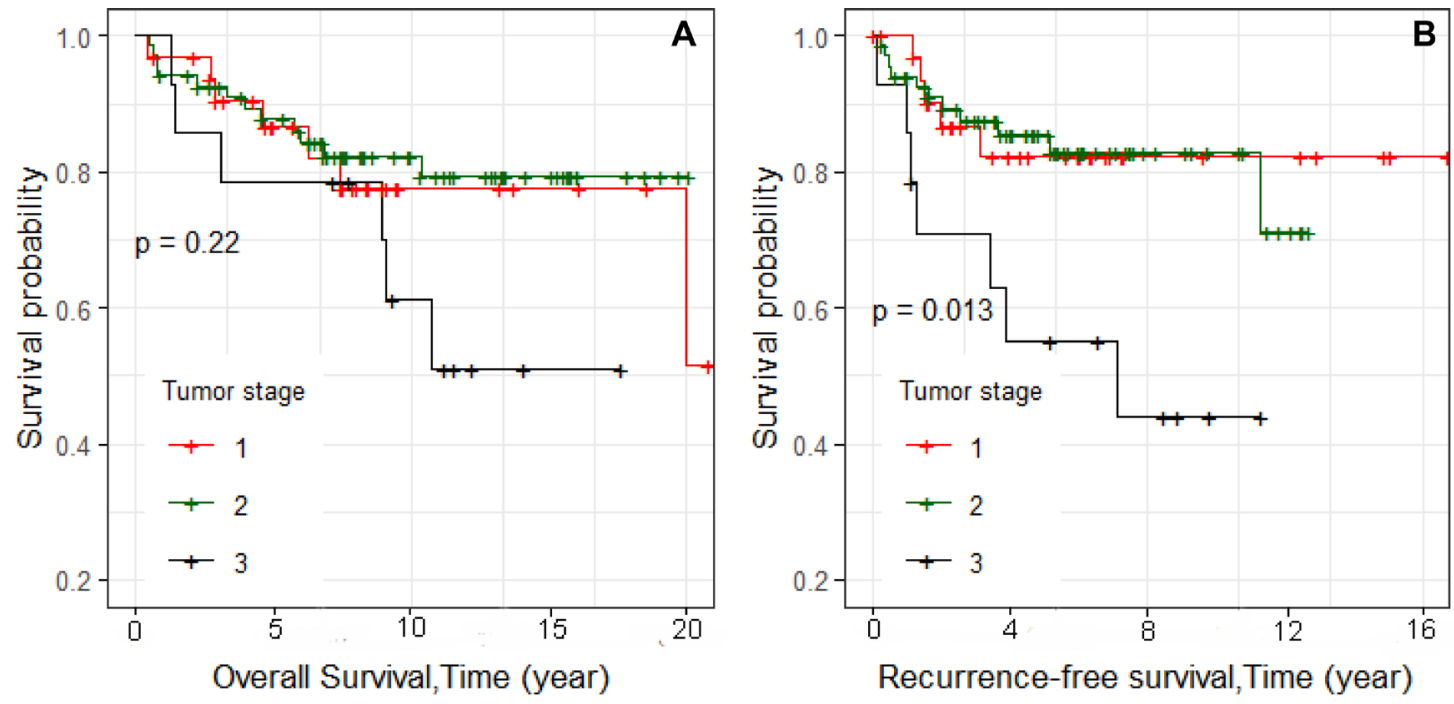

Figure 2: Kaplan-Meier analyses of overall and recurrence-free survival and stage for 115 TNBC cases (Figure 2 A and $2 \mathrm{~B}$, respectively). 

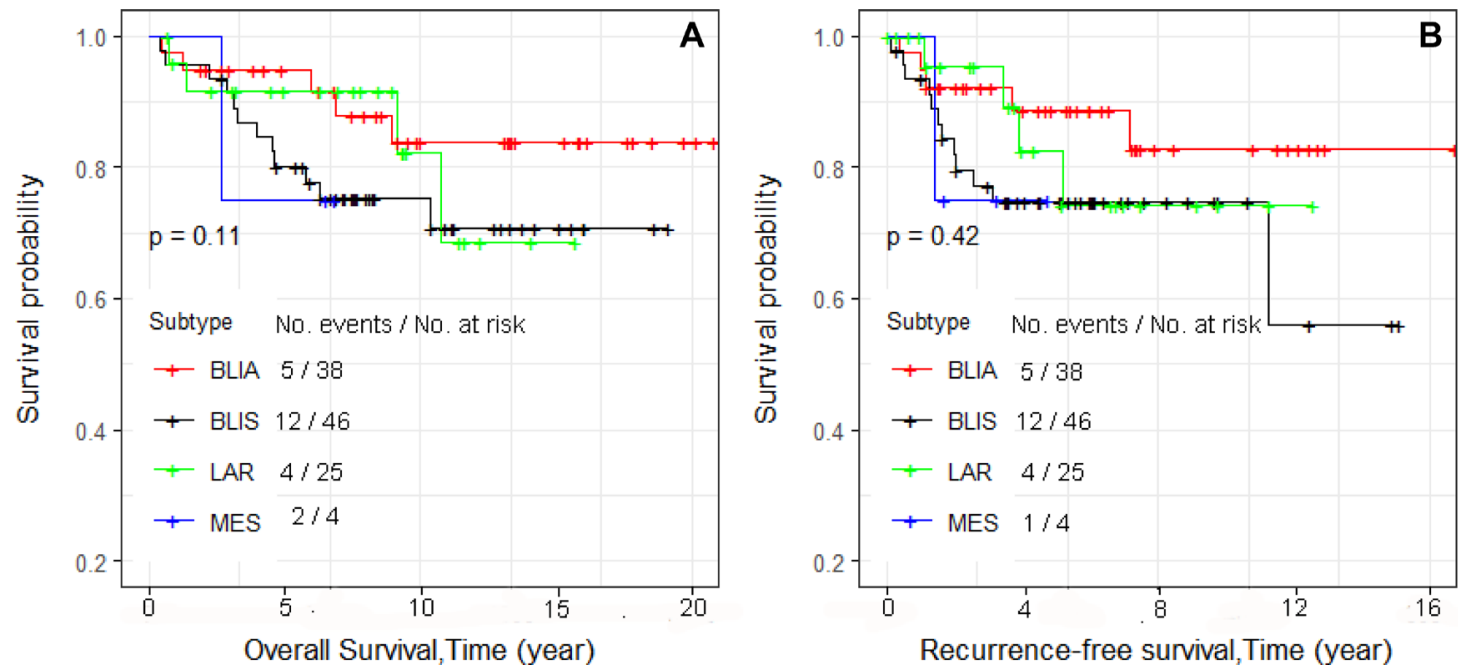

Figure 3: Kaplan-Meier analyses of overall survival (A) and recurrence-free survival (B) and four subtypes for 113 TNBC patients. Number of patient at risk and cumulative number of events over a time period were summarized in each plot.
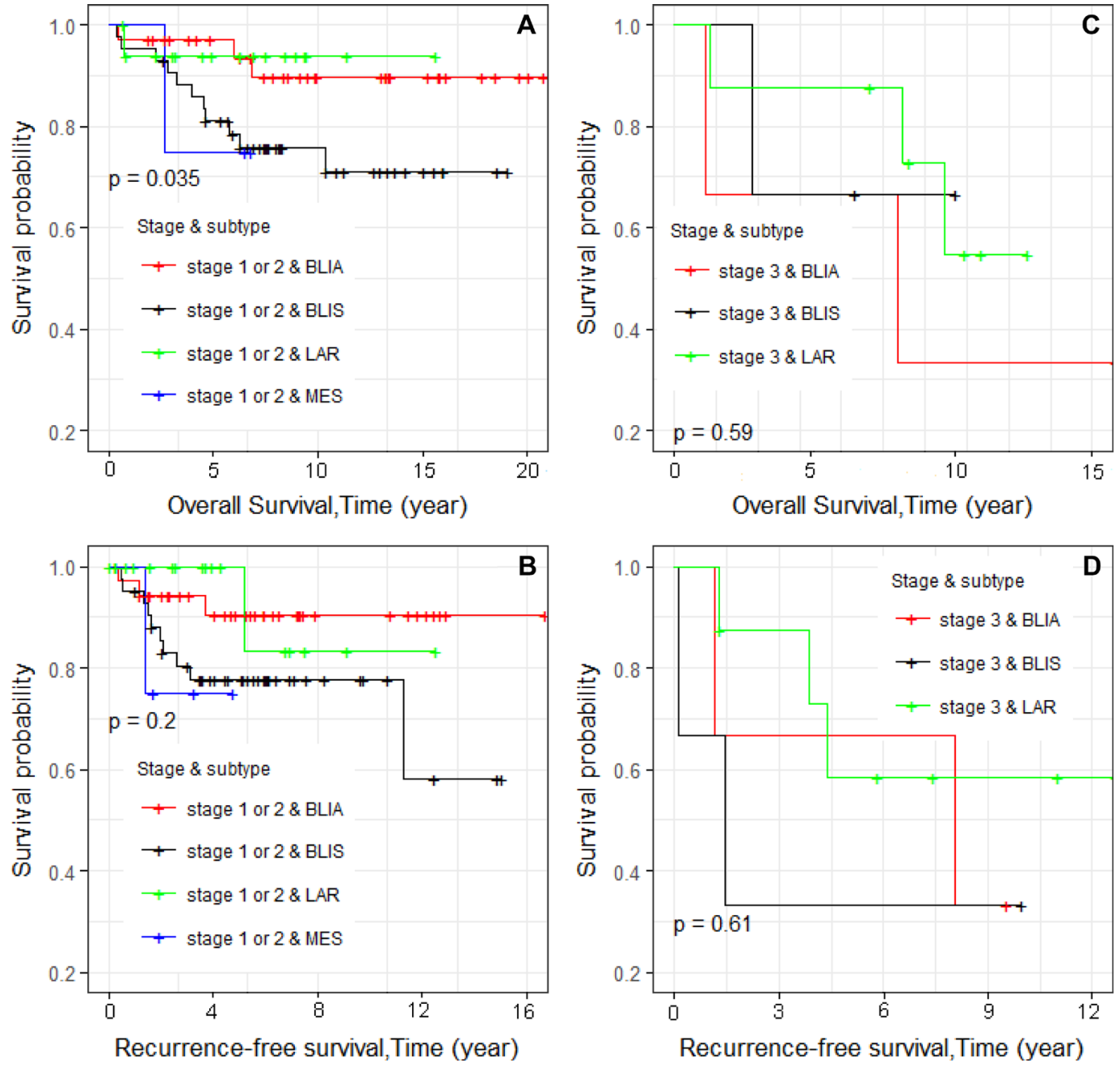

Figure 4: Kaplan-Meier analyses of overall survival and recurrence-free survival and tumor subtype for 113 TNBC patients stratified by tumor stages 1 and 2 and tumor stage 3 (A-D, respectively). 
Table 3: Cox-proportional hazard models of association of molecular subtypes with recurrence-free survival and overall survival

\begin{tabular}{|c|c|c|c|c|}
\hline Survival & Comparison & Adjusted HR* & 95\% CI for HR & $p$ value \\
\hline \multirow[t]{7}{*}{ Recurrence-free survival } & BLIA & 1.000 & & \\
\hline & BLIS & 2.951 & $0.871-9.990$ & 0.082 \\
\hline & LAR & 0.253 & $0.049-1.310$ & 0.101 \\
\hline & MES & 6.006 & $0.542-66.550$ & 0.144 \\
\hline & BLIS & 1.000 & & \\
\hline & LAR & 0.086 & $0.017-0.420$ & 0.002 \\
\hline & MES & 2.036 & $0.221-18.740$ & 0.531 \\
\hline \multirow[t]{7}{*}{ Overall survival } & BLIA & 1.000 & & \\
\hline & BLIS & 2.611 & $0.788-8.660$ & 0.115 \\
\hline & LAR & 0.192 & $0.037-0.990$ & 0.049 \\
\hline & MES & 4.668 & $0.550-39.910$ & 0.159 \\
\hline & BLIS & 1.000 & & \\
\hline & LAR & 0.073 & $0.015-0.354$ & 0.001 \\
\hline & MES & 1.787 & $0.257-12.426$ & 0.557 \\
\hline
\end{tabular}

"HR, hazard ratio; CI, confidence interval. Cox proportional hazard regression models were used to estimate association between a subtype and overall and recurrence-free survival, including age at diagnosis, race/ethnicity, and tumor stage as covariates. Smoothed plots of weighted Schoenfeld residuals were used to test the assumption of proportionality.

Table 4: Association of specific subtype with race and ethnicity

\begin{tabular}{lcccccccc}
\hline & \multicolumn{7}{c}{ Subtype comparisons, count (\%) } \\
\hline Race and ethnicity & Yes-LAR & No-LAR & $\boldsymbol{p}$ value & coefficient & Yes-BLIS & No-BLIS & $\boldsymbol{p}$ value & coefficient \\
\hline White & $9(27.3)$ & $24(72.7)$ & 0.49 & 0.27 & $12(36.4)$ & $21(63.6)$ & 0.99 & -0.01 \\
Hispanic & $6(12.8)$ & $41(87.2)$ & 0.10 & -0.67 & $25(53.2)$ & $22(46.8)$ & 0.03 & 0.68 \\
African American & $2(16.7)$ & $10(83.3)$ & 0.55 & -0.36 & $5(41.7)$ & $7(58.3)$ & 0.65 & 0.22 \\
Asian & $8(38.1)$ & $13(61.9)$ & 0.06 & 0.76 & $4(19.1)$ & $17(80.9)$ & 0.05 & -0.89 \\
Total & $25(22.1)$ & $88(77.9)$ & & & $46(40.7)$ & $67(59.3)$ & & \\
\hline
\end{tabular}

The binary logistic regression model was used to assess relationship between subtype-LAR or BLIS (yes or no, response variable) and race and ethnicity (explanatory variable); the coefficient $p$-values is based on the Wald test.

In this study, a much lower percentage of MES subtype was observed (3.5\% versus $17.8 \%$ in Burstein et al. [11]), possibly because of the more stringent isolation criteria used in our study.

The comparative analyses of LAR of the Lehman and Burstein signatures had significant overlap. We also found that correlation coefficients for LAR subtype were high, and there was clear definition of the subtype with the signature (Supplementary Figure 1). In contrast to the LAR signature, the BLIA and BLIS signatures were less distinct (Supplementary Figure 1). If we define a mixed subtype as one where the measured difference of the top-two Spearman correlation values in a sample is less than 0.1 [16], then 29 of $115(25 \%)$ samples would be classified as mixed BLIA-BLIS subtypes, and another 9 of $115(8 \%)$ samples would have other mixed subtypes (see
Supplementary Table 2). There were fewer mixed subtypes among the Asian cases compared to the other groups as well as a significantly greater correlation difference between the top two subtypes $(p<0.05)$ (Supplementary Table 2 and Supplementary Figure 2). The data of Burstein et al. and of Lehman et al. were similar to our results for mixed subtypes in that 27 of the 114 Burstein validation samples $(23.7 \%)$ and 76 of the 243 Lehman validation samples $(31.3 \%)$ were a mix of two subtypes based on this cut-off. This suggests that tumors are heterogeneous and contain multiple subtypes and/or that the subtype signatures may require further refinement. Interestingly, we found that when including the BLIS-BLIA mixed subtype in Kaplan-Meier analyses, this mixed group subtype fell between the BLIA and BLIS subtypes for recurrence-free survival and overall survival (see Supplementary Figure 3). 
The recent development of molecular classifiers of TNBC presents a real opportunity to improve therapies and therapeutic choices. Potential subtype-specific therapeutic targets previously were identified based on the unique gene expression profiles [11] of their 80-gene signature. In order to potentially improve the signature, we added an additional 11 genes from a methylome study which classified TNBCs into prognostic clusters, and used NanoString assays to measure expression. This technology is robust and accurate for RNA from FFPE samples [17, 18]. Because it is hybridization-based, it does not require reverse transcription of mRNA and cDNA amplification thus reducing amplification bias; and because it is focused on a small number of genes rather than an expression array, it is more cost effective.

This study was designed to study the association of molecular subtypes of TNBCs and disparities in survival across races and ethnicities. We found that TNBC samples from Hispanic and Asian women had significantly different proportions of BLIS and LAR compared to the average proportion of corresponding subtype across all four groups. These differences in the distributions of TNBC subtypes may reflect true biologic/genetic differences by race and ethnicity. There may be better signatures to define subtypes in different racial and ethnic populations as the signatures were originally developed using TNBC samples from primarily White women. We will need to perform global RNA-seq in the future to identify if there are additional biomarkers that may separate Hispanic and Asian TNBC subtypes. Another possibility is that although our sample size is reasonable, it is divided among 4 groups of different race and ethnicity. This may make group sizes too small to have adequate power to detect all the differences. That appears to be the case for the African-American TNBC cases, which had a genetic signature more similar to the Hispanic cases, but the sample size did not have sufficient power to discern statistical differences.

This study is one of the first to conduct molecular subtyping of TNBC comparing Whites, Hispanics, Asians, and African Americans; it fills a gap of knowledge in how molecular subtypes of TNBC may be used to improve tumor-biology-driven prognosis of TNBC in these underserved and understudied populations. In multivariate analysis accounting for age and stage at diagnosis and race/ethnicity, our results show that TNBC subtype BLIS is associated with worse survival and LAR with the best survival (Table 3). This result was consistent with a study comparing AR+/epidermal growth factor (EGFR) - tumors (the LAR subtype), in which they reported that LAR had the best prognosis compared to the basal TNBC subtype (defined as AR-/EGFR+) [19]. In univariate Kaplan-Meier analyses, our results were consistent with those reported by Burstein et al. [11]; although not statistically significant, we found that BLIS had the worst survival and BLIA the best survival compared to the other subtypes. Our results differ from those reported by
Lehmann et al. [14] where they observed non-significantly worse survival for LAR. There likely are several reasons for this inconsistency: 1) these are chance differences as their findings were not statistically significant; 2) we conducted multivariate analyses adjusting for age at diagnosis and stage of the cancer; both factors that were significantly associated with subtype (Table 2); 3) LAR is the only subtype that overlaps between the Burstein et al. [11] and Lehmann et al. subtypes [14]; 4) we excluded women who were treated with neoadjuvant therapy - pathological complete response for LAR is worse than for BL1 which would reflect in Kaplan-Meier analyses. The Asian women in the study had significantly lower numbers of BLIS and higher numbers of LAR, and they showed the best overall survival which is consistent with what has been previously reported for survival [13]. From a public health perspective, the sub-typing of TNBCs may facilitate understanding of the heterogeneity of the TNBCs and provide a foundation for developing subtype-specific therapies and better predictors of TNBC prognosis for all races and ethnicities.

\section{MATERIALS AND METHODS}

\section{Identification and selection of patients}

Paraffin blocks from formalin-fixed primary breast cancer tissue specimens were obtained from the City of Hope Biorepository through an Institutional Review Board approved protocol. Patients were selected on the basis of self-reported ethnicity and race, TNBC diagnosis was from January 2000 through July 2016, and tumor tissue availability. Tissue samples were procured from 115 patients with TNBCs who had not had neo-adjuvant therapy. Our primary focus was Hispanic Caucasian women primarily from Mexico, referred to as Hispanic. We then selected Asians (East Asians, primarily Han Chinese) and African Americans and then Whites (nonHispanic Caucasians). Data on family history of breast cancer, age, pathology stage, receptor status, chemotherapy regimens, time to recurrence, and cause and date of death (if occurred) were captured through chart review.

\section{RNA preparation}

A surgical pathologist identified foci with greater than $80 \%$ tumor on hematoxylin and eosin stained slides from formalin-fixed paraffin-embedded (FFPE) tissue. For RNA extraction, the corresponding areas were microdissected from 10-micron unstained sections. The specific area(s) from the unstained sections were then dissected for extraction of RNA. RecoverAll ${ }^{\mathrm{TM}}$ Total Nucleic Acid Isolation kit (Ambion) was used to extract total RNA following the manufacturer's instructions. RNA was quantified by fluorometry using Quant-iT RNA BR Assay Kit. Quality was assessed using the 2100 Bioanalyzer (Agilent Technologies). 


\section{Gene expression assays}

For each FFPE sample, 250 ng of extracted RNA was isolated for analysis by nCounter Gene Expression Codesets (NanoString Technologies). For gene expression profiling, we measured expression of 99 genes; this set included the 80 genes in the expression signature in Burstein et al. [11], 12 genes from a methylome study that identified prognostic clusters (1 overlaps with Burstein gene signature set) [12], and 8 housekeeping genes (ACTB, GUSB, MRPL19, PSMC4, PUM1, RPLP0, SF3A1, and $T F R C$ ) for normalization from PAM50 (Prosigna). In addition to the 115 patient samples described above, an additional 5 TNBC samples with accompanying Affymetrix expression array data were tested as known control subtypes to ensure that this first use of NanoString technology to define subtypes faithfully recapitulated the Affymetrix expression signatures. The $\mathrm{COH}$ Molecular Diagnostic Lab processed the RNA samples and produced files with raw counts for each gene in each sample.

\section{Data analysis}

nCounter raw data were background assessed and quality checked using nCounter internal negative and positive controls by following the data analysis workflow from NanoString Technologies. Using nSolver Analysis Software, the absolute transcript abundance was determined for each sample by further normalizing expression with the housekeeping genes listed above. Log2 transformation of normalized gene expression data was used for TNBC subtype classification. We used the gene expression data (GSE76124) from the Burstein et al. [11] dataset, which included 84 samples in the training set and 114 samples in the validation set. Because we added 11 genes to the gene signature of Burstein et al. [11] (described above under Gene Expression assays), PAM method $[20,21]$ was used to select the optimal subset of genes to classify the four TNBC subtypes BLIA, BLIS, LAR, and MES. From this analysis, a gene set was selected, and quantile expression centroids of the four subtypes of TNBC were calculated from the 84 training samples.

To account for batch effects between the Burstein training data set [11] and our test data, the addon batch adjustment technique $[22,23]$ was used to adjust our test data to the Burstein training data; the prediction rules generated from the training data were used to predict subtypes in our data. Principal component analysis (PCA) was used to assess the effect of the addon batch adjustment (see Supplementary Figure 4). Before the batch adjustment, PCA results showed spatial separation of the training data from our test data (Supplementary Figure 4A); after batch adjustment, the two data sets were spatially aligned (Supplementary Figure 4B). Then one of four TNBC subtypes was assigned to each of our 120 samples (5 control samples with Affymetrix gene expression data and 115 test samples) based on the strongest Spearman correlation between sample expression and the 77-gene subtype centroid signatures (see Supplementary Table 2). The subtype was assigned based on the highest Spearman correlation coefficient regardless of the difference between the top two Spearman correlation values. For the five control samples, two were assigned to the BLIA subtype, two to the BLIS subtype, and one to the MES subtype, all consistent with the subtypes assigned using Affymetrix gene expression data.

\section{Statistical analyses}

Patient and disease characteristics included: a) age, stage at diagnosis, race or ethnicity, and family history of breast cancer; b) overall survival defined as time from the date of surgery to death from any cause; and c) recurrencefree survival defined as time from date of surgery to breast cancer recurrence. Fisher's exact test was used to test the association between subtypes and categorical clinical variables including tumor stage, race, tumor grade, positive lymph node, and family history of breast cancer (yes/no). Binary logistic regression analysis was used to assess the direction and strength of association between a specific subtype (response variable; yes or no) and race or ethnicity (explanatory variable).

For recurrence-free survival, time was censored at death if cause of death was not from breast cancer or at last contact if the patient was still alive at last contact date. For overall survival, time was censored at last contact date. For univariate analysis, overall and recurrence-free survival curves were generated by Kaplan-Meier method, and a log-rank test was employed to assess survival difference. For multivariate analysis, Cox regression models were used to estimate association between a subtype and overall and recurrence-free survival, including age at diagnosis, race or ethnicity, and tumor stage as covariates. Smoothed plots of weighted Schoenfeld residuals generated by the cox. zph function of survival package in $\mathrm{R}$ were used to test the assumption of proportionality for all predictors in the Cox model [24]; results are shown in Supplementary Figure 5.

\section{Abbreviations}

TNBC: triple negative breast cancer; HR: hazard ratio; CI: confidence interval; BLIA: basal-like immune activated; BLIS: basal-like immunosuppressed; LAR: luminal androgen receptor; MES: mesenchymal; TCGA: The Cancer Genome Atlas; NMF: non-negative matrix factorization.

\section{Author contributions}

SLN, YY, and YCD designed experiments, LS, YCD, SW, CW and SLN performed experiments and analyzed data; YCD and SLN wrote the manuscript, YY 
and JM provided critical feedback, all authors edited and reviewed the manuscript.

\section{ACKNOWLEDGMENTS}

We thank Chris Gandhi for assistance in editing the manuscript for clarity.

\section{CONFLICTS OF INTEREST}

The authors have no conflicts of interest to disclose.

\section{FUNDING}

This project was supported by the Morris and Horowitz Families Professorship (SLN) and a City of Hope Women's Cancer Program pilot grant. Research reported in this publication included work performed in the Integrative Genomics and Pathology cores supported by the National Cancer Institute of the National Institutes of Health under award number P30CA033572.

\section{REFERENCES}

1. Dent R, Trudeau M, Pritchard KI, Hanna WM, Kahn HK, Sawka CA, Lickley LA, Rawlinson E, Sun P, Narod SA. Triple-negative breast cancer: clinical features and patterns of recurrence. Clin Cancer Res. 2007; 13:4429-34. https:// doi.org/10.1158/1078-0432.CCR-06-3045.

2. Bulut N, Kilickap S, Sari E, Altundag K. Response to taxanes in triple negative breast cancer. Cancer Chemother Pharmacol. 2008; 63:189. https://doi.org/10.1007/ s00280-008-0717-7.

3. Howlader N, Altekruse SF, Li CI, Chen VW, Clarke CA, Ries LA, Cronin KA. US incidence of breast cancer subtypes defined by joint hormone receptor and HER 2 status. J Natl Cancer Inst. 2014; 106:106. https://doi.org/10.1093/ jnci/dju055.

4. Bauer KR, Brown M, Cress RD, Parise CA, Caggiano V. Descriptive analysis of estrogen receptor (ER)-negative, progesterone receptor (PR)-negative, and HER2-negative invasive breast cancer, the so-called triple-negative phenotype: a population-based study from the California cancer Registry. Cancer. 2007; 109:1721-28. https://doi. org/10.1002/cncr.22618.

5. Hines LM, Risendal B, Byers T, Mengshol S, Lowery J, Singh M. Ethnic disparities in breast tumor phenotypic subtypes in Hispanic and non-Hispanic white women. J Womens Health (Larchmt). 2011; 20:1543-50. https://doi. org/10.1089/jwh.2010.2558.

6. Stark A, Kleer CG, Martin I, Awuah B, Nsiah-Asare A, Takyi V, Braman M, Quayson SE, Zarbo R, Wicha M, Newman L. African ancestry and higher prevalence of triple-negative breast cancer: findings from an international study. Cancer. 2010; 116:4926-32. https://doi.org/10.1002/cncr.25276.

7. Stead LA, Lash TL, Sobieraj JE, Chi DD, Westrup JL, Charlot M, Blanchard RA, Lee JC, King TC, Rosenberg CL. Triple-negative breast cancers are increased in black women regardless of age or body mass index. Breast Cancer Res. 2009; 11:R18. https://doi.org/10.1186/bcr2242.

8. Abramson VG, Lehmann BD, Ballinger TJ, Pietenpol JA. Subtyping of triple-negative breast cancer: implications for therapy. Cancer. 2015; 121:8-16. https://doi.org/10.1002/ cncr.28914.

9. Lehmann BD, Bauer JA, Chen X, Sanders ME, Chakravarthy AB, Shyr Y, Pietenpol JA. Identification of human triple-negative breast cancer subtypes and preclinical models for selection of targeted therapies. J Clin Invest. 2011; 121:2750-67. https://doi.org/10.1172/JCI45014.

10. Masuda H, Baggerly KA, Wang Y, Zhang Y, GonzalezAngulo AM, Meric-Bernstam F, Valero V, Lehmann BD, Pietenpol JA, Hortobagyi GN, Symmans WF, Ueno NT. Differential response to neoadjuvant chemotherapy among 7 triple-negative breast cancer molecular subtypes. Clin Cancer Res. 2013; 19:5533-40. https://doi. org/10.1158/1078-0432.CCR-13-0799.

11. Burstein MD, Tsimelzon A, Poage GM, Covington KR, Contreras A, Fuqua SA, Savage MI, Osborne CK, Hilsenbeck SG, Chang JC, Mills GB, Lau CC, Brown PH. Comprehensive genomic analysis identifies novel subtypes and targets of triple-negative breast cancer. Clin Cancer Res. 2015; 21:1688-98. https://doi.org/10.1158/1078-0432. CCR-14-0432.

12. Stirzaker C, Zotenko E, Song JZ, Qu W, Nair SS, Locke WJ, Stone A, Armstong NJ, Robinson MD, Dobrovic A, Avery-Kiejda KA, Peters KM, French JD, et al. Methylome sequencing in triple-negative breast cancer reveals distinct methylation clusters with prognostic value. Nat Commun. 2015; 6:5899. https://doi.org/10.1038/ncomms6899.

13. Breast Cancer Facts and Figures 2013-2014. American Cancer Society. 2013.

14. Lehmann BD, Jovanović B, Chen X, Estrada MV, Johnson KN, Shyr Y, Moses HL, Sanders ME, Pietenpol JA. Refinement of Triple-Negative Breast Cancer Molecular Subtypes: Implications for Neoadjuvant Chemotherapy Selection. PLoS One. 2016; 11:e0157368. https://doi. org/10.1371/journal.pone.0157368.

15. Pareja F, Geyer FC, Marchiò C, Burke KA, Weigelt B, ReisFilho JS. Triple-negative breast cancer: the importance of molecular and histologic subtyping, and recognition of lowgrade variants. NPJ Breast Cancer. 2016; 2:16036. https:// doi.org/10.1038/npjbcancer.2016.36.

16. Cheang MC, Chia SK, Voduc D, Gao D, Leung S, Snider J, Watson M, Davies S, Bernard PS, Parker JS, Perou CM, Ellis MJ, Nielsen TO. Ki67 index, HER2 status, and prognosis of patients with luminal B breast cancer. J Natl Cancer Inst. 2009; 101:736-50. https://doi.org/10.1093/ jnci/djp082.

17. Geiss GK, Bumgarner RE, Birditt B, Dahl T, Dowidar N, Dunaway DL, Fell HP, Ferree S, George RD, Grogan T, James JJ, Maysuria M, Mitton JD, et al. Direct multiplexed measurement of gene expression with color-coded probe pairs. Nat Biotechnol. 2008; 26:317-25. https://doi. org/10.1038/nbt1385.

18. Malkov VA, Serikawa KA, Balantac N, Watters J, Geiss G, Mashadi-Hossein A, Fare T. Multiplexed measurements of gene signatures in different analytes using the Nanostring nCounter Assay System. BMC Res Notes. 2009; 2:80. https://doi.org/10.1186/1756-0500-2-80. 
19. Astvatsaturyan K, Yue Y, Walts AE, Bose S. Androgen receptor positive triple negative breast cancer: Clinicopathologic, prognostic, and predictive features. PLoS One. 2018; 13:e0197827. https://doi.org/10.1371/ journal.pone.0197827.

20. Blagus R, Lusa L. Improved shrunken centroid classifiers for high-dimensional class-imbalanced data. BMC Bioinformatics. 2013; 14:64. https://doi. org/10.1186/1471-2105-14-64.

21. Tibshirani R, Hastie T, Narasimhan B, Chu G. Diagnosis of multiple cancer types by shrunken centroids of gene expression. Proc Natl Acad Sci U S A. 2002; 99:6567-72. https:// doi.org/10.1073/pnas.082099299.

22. Hornung R, Boulesteix AL, Causeur D. Combining location-and-scale batch effect adjustment with data cleaning by latent factor adjustment. BMC Bioinformatics. 2016; 17:27. https://doi.org/10.1186/s12859-015-0870-z.

23. Johnson WE, Li C, Rabinovic A. Adjusting batch effects in microarray expression data using empirical Bayes methods. Biostatistics. 2007; 8:118-27. https://doi.org/10.1093/ biostatistics/kxj037.

24. Grambsch PM, Therneau TM. Proportional hazards tests and diagnostics based on weighted residuals. Biometrika. 1994; 81:515-26. https://doi.org/10.1093/biomet/81.3.515. 